\title{
Interannual-to-interdecadal variability in PMIP simulations at the local to global scale
}

\author{
Kira Rehfeld ${ }^{1,2}$ and Josephine Brown ${ }^{3}$
}

\author{
Here, we outline recent insights into interannual to decadal variability of Earth's surface climate based on PMIP \\ experiments and comparison with future climate simulations. These studies have provided new perspectives on large- \\ scale changes of surface climate, low- and high-latitude modes of variability, and internally versus externally forced \\ variability.
}

\section{Climate variability in past, present and future}

Interannual to decadal variability of surface climate variables arises through internal dynamics in the atmosphere-hydrospherebiosphere-cryosphere system driven by the incoming solar radiation. External forcing impacts the climate system on multiple timescales. Constant adjustment of the Earth's energy budget through feedbacks and dynamics lead to changes in the global mean temperature, regional patterns, and fluctuations around the regional mean-in other words, to climate variability. Variations in the Earth's orbit (104-103 years) change the seasonal distribution of insolation quasi-periodically, while changes in solar luminosity $\left(10^{3}-10^{1}\right.$ years) modulate the overall energy input to the system. At the same time, explosive volcanic eruptions stochastically perturb the system on seasonal to interannual timescales.

Equilibrium simulations from PMIP3 and PMIP4 allow us to examine the response of the climate system to different orbital insolation, topography, ice-sheet configurations and greenhouse-gas concentrations (Braconnot et al. 2012; Kageyama et al. 2018). Simulations for the last (pre-industrial) millennium (Jungclaus et al. 2017) have been used frequently to test the impact of solar and volcanic forcing on climate at interannual to centennial timescales. The PMIP4 working group "Past2Future: Insights from a constantly varying past" (pmip4.Isce.ipsl.fr/ doku.php/wg:ptof:index) aims to improve our general understanding of climate stability through multi-model analyses of a range of climate states, focusing on large-scale patterns of change and internal modes of variability. It builds on the efforts of the PMIP3 working groups "Past to future" and "Paleovar".

\section{Large-scale changes in simulated climate variability}

The PMIP last millennium experiments have been key to improving our understanding of the role of volcanism and solar variability in driving climate variations. Studies incorporating both proxy and model data to assess mechanisms, blended reconstructions, and impacts on society draw on the considerable overlap between observations, and the dense proxy networks fostered by different PAGES working groups.

One key insight is that current climate models show too little variability locally, at individual observation locations. This was shown by Laepple and Huybers (2014), who investigated ocean surface temperature variability in CMIP5/PMIP3 last millennium simulations. At interannual timescales (2-5 years), no systematic offset between simulated regional temperature variability and observations could be found. However on decadal to multicentennial timescales they observed a progressive increase in the underestimation of variability in gridboxscale model surface ocean temperatures. On the other hand, the global mean simulated and reconstructed/observed temperature variability on interannual to multidecadal timescales are of similar magnitude over the last millennium (Laepple and Huybers 2014; Parsons et al. 2020) and the Common Era
(PAGES 2k Consortium 2019). This consistency of simulated and reconstructed variability at the global scale, despite the lack of modeled regional variability at decadal and longer timescales, remains unexplained.

PMIP experiments targeting time periods prior to the late Holocene include a range of boundary conditions such as the land-sea mask, orbital parameters, greenhouse gas concentrations, and ice-sheet distribution. These experiments generally do not consider forcing on interannual to centennial timescales by changes in solar luminosity or explosive volcanism, as proxy-based reconstructions do not yet exist (and may not be possible given archive and proxy uncertainties). This implies that changes in interannual to multidecadal variability in these equilibrium simulations reflect the internal dynamical response of the climate system to the boundary conditions.

Interannual to multidecadal variability changes systematically across equilibrium simulations for the LGM, the mid-Holocene, and for idealized warming scenarios (abrupt4xCO2 and 1pctCO2; Taylor et al. 2012). Figure 1 illustrates the large-scale and mirroring changes between interannual to decadal temperature and precipitation variability in the LGM and 1 pctCO2 cases. At the global scale, the colder climate is characterized by more variability in temperature and less in precipitation in most regions. The warming scenario is associated with increasing temperature and precipitation variability across the tropics and subtropics. Changes follow a contrasting land-sea pattern.
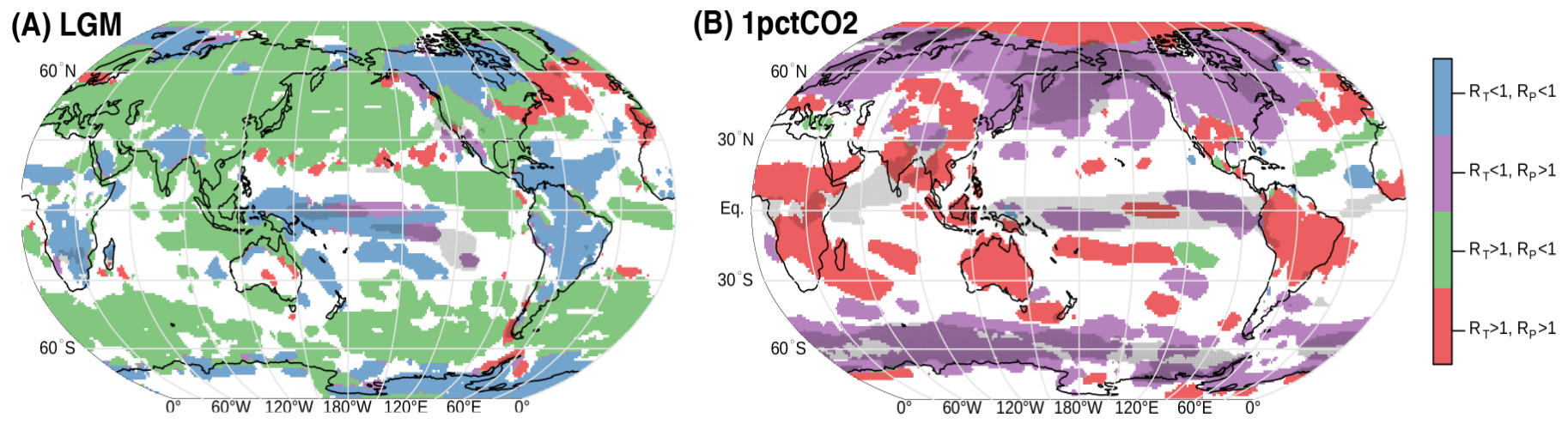

Figure 1: Temperature and precipitation variance change systematically with global mean temperature. Variance ratios $\left(R=\sigma_{\text {scen }} / \sigma_{p i}\right.$ where $\sigma_{\text {pi }}$ is the standard deviation of the preindustrial control simulation and $\sigma_{\text {scen }}$ is the variance of $\{\mathrm{LGM}, 4 \times C O 2\}$ ) were calculated from each CMIP5/CMIP6/PMIP3 model on (A) the last 50 years of the LGM simulations and (B) years 101-150 of the 1 pctCO2 increase scenarios and compared to the final 50 years of the pre-industrial simulations. All simulations were linearly detrended and variance ratios were averaged. Colors classify regions with concurrent changes in temperature $\left(R_{T}\right)$ and precipitation $\left(R_{p}\right)$ variability. Changes of less than $5 \%$ are masked as white. Black shading indicates an increase in total precipitation by more than $0.4 \mathrm{~mm} /$ day in the annual mean. Visualization by J. Bühler based on data from Rehfeld et al. (2020). 


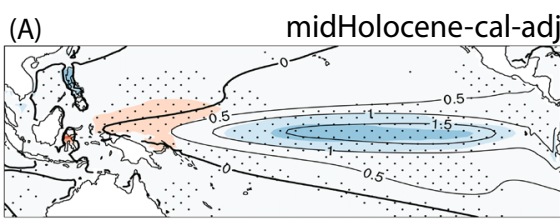

(B)

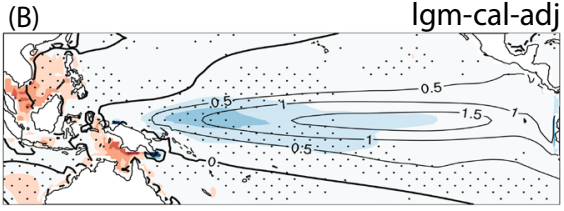

(C) lig127k-cal-adj

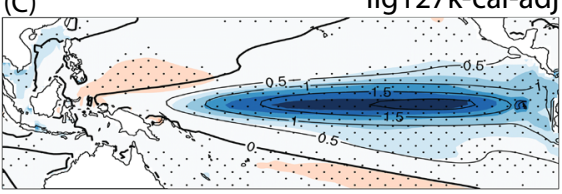

(D) 1 pctCO2

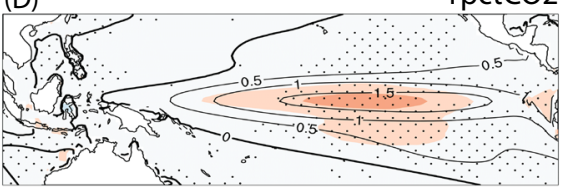

(E) abrupt $4 \times \mathrm{CO} 2$

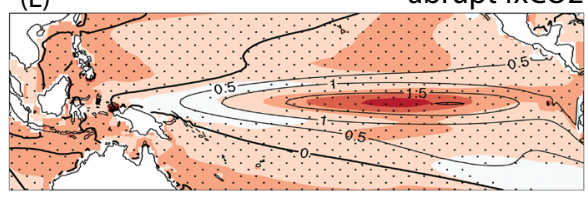

$\begin{array}{llllllllll}-0.5 & -0.4 & -0.3 & -0.2 & -0.1 & 0.1 & 0.2 & 0.3 & 0.4 & 0.5\end{array}$

Figure 2: Changes in SST variability associated with ENSO for PMIP and CMIP experiments. The ensemble mean difference between the SST composites in each model during El Niño minus La Niña (defined as \pm 1 standard deviation) in the (A) midHolocene, (B) lgm, (C) lig127k, (D) 1 pctCO2 and $(\mathbf{E})$ abrupt $4 \times \mathrm{CO} 2$ experiments minus the same pattern for the piControl simulations is shown. The ensemble mean ENSO SST patterns in the piControl simulations are shown as black contours. Stippling indicates that more than two-thirds of the ensemble members agree on the sign of the change. Modified from Brown et al. (2020).

Spectral analysis showed that these general patterns hold from seasonal to multidecadal timescales across the PMIP3/CMIP5/CMIP6 model ensemble (Rehfeld et al. 2020).

\section{Changes in modes of climate variability}

Many studies have investigated changes in the El Niño Southern Oscillation (ENSO) characteristics in PMIP experiments. Zheng et al. (2008) compared multiple models for paleoclimate (PMIP2 LGM, mid-Holocene experiments) and future simulations, finding relationships between the tropical Pacific mean state and ENSO amplitude, as well as a reduced mid-Holocene ENSO variability. An and Choi (2014) compared ENSO in PMIP2 and PMIP3 mid-Holocene experiments and found a significant reduction in ENSO amplitude for PMIP2 models but only a marginal reduction in PMIP3 models due to competing processes, with weakened airsea coupling leading to suppressed ENSO but weakening of the annual cycle over the tropical eastern Pacific supporting intensified ENSO.

Using an ensemble of PMIP3 and PMIP4 mid-Holocene experiments, Brown et al.
(2020) found a consistent reduction in ENSO amplitude of $7 \%$ for PMIP3 models and $10 \%$ for PMIP4 models relative to pre-industrial ENSO amplitude. Comparison of midHolocene proxy records and PMIP3 simulations showed that models underestimated the reduction in ENSO amplitude compared with proxy reconstructions (Emile-Geay et al. 2016). Investigation of PMIP4 last interglacial experiments showed a stronger reduction in ENSO amplitude of around $20 \%$, consistent with the larger seasonal insolation anomalies than the mid-Holocene experiments (Brown et al. 2020).

\section{Simulations of ENSO in PMIP2 and PMIP3}

LGM experiments showed a range of changes in ENSO characteristics (MassonDelmotte et al. 2013). PMIP4 LGM simulations show no significant change in ENSO amplitude but reduced variability in the western Pacific SST variability, indicating a spatial shift in the ENSO pattern (Brown et al. 2020). Examination of ENSO in past cold and warm climates (as shown in Fig. 2) can provide insights into the relationship between changes in the mean state and ENSO variability (Saint-Lu et al. 2015), and may assist in constraining projections of future ENSO change.

Across the PMIP3/CMIP5/CMIP6 ensemble, ENSO indices showed increasing variability with warming, but the changes were not significant given the large intermodel spread (Rehfeld et al. 2020). Similarly, some other interannual to multidecadal modes of variability showed systematic, but weak, changes in variability with increasing global mean temperature across the PMIP3 ensemble. This included the boreal winter North Atlantic Oscillation and the Northern Annular Mode (weakly positive), amongst others. The Atlantic meridional and zonal modes showed decreasing variability with warming. For many of the other proposed modes of variability the integration length (typically 50-100 years) was insufficient to as sess whether or not systematic changes are expected to occur with regional or global warming.

This general perspective of reduced temperature variability with warming at the global scale is consistent with the direction of temperature variability changes from a multi-proxy study targeting multicentennial to millennial timescales (Rehfeld et al. 2018). Proxy-based confirmation on shorter timescales will, however, require reduced age uncertainties, removal of confounding effects due to other climate variables, the environment, or the archive structure, and an expansion of the high-resolution proxy network.

\section{Challenges and Perspectives}

A series of interconnected challenges need to be tackled regarding the paleoclimate record and paleoclimate modeling, in order to further enhance our understanding of changes in interannual to multidecadal variability. Firstly, this entails testing the impact of centennial- to millennial-scale variations in the mean state on variability at these shorter timescales. On the modeling side this requires the incorporation of nonstationary elements such as ice sheets, biogeochemistry, and land surface processes, but also a reasonable understanding of the nature of the variability of the glacial ocean circulation. This could be facilitated by considering an ensemble of models of different complexity together to assess stabilizing and destabilizing feedbacks of low frequency changes on interannual variability.

Spatio-temporal shifts in modes operating on interannual to decadal timescales can be expected to occur with warming. This has been extensively studied for ENSO, where shifts in the frequency of different "flavors" or spatial patterns of ENSO may occur. Examination of PMIP mid-Holocene simulations suggested changes in the occurrence of Central Pacific versus Eastern Pacific events (e.g. An and Choi 2014; Emile-Geay et al. 2016). Shifts in the spatial pattern of ENSO in past climates therefore need to be considered when carrying out model-proxy comparisons. To evaluate the simulated variability, especially in pre-Holocene time periods, the proxy network needs further consolidation in time and space, in order to assess signal-to-noise ratios and distinguish model deficiencies (e.g. underestimated SST variability) from archive noise (e.g. from bioturbation, intermittency, or aliasing). The comparability of modeled and reconstructed signals could further be improved by forward modeling of tracer species (e.g. water isotopologs) in collaboration with PMIP/CMIP experiments, longer model integrations, and the inclusion of solar and volcanic forcings in experiments.

\section{AFFILIATIONS}

'Geo- und Umweltforschungszentrum, Tübingen University

Institute of Environmental Physics, Heidelberg University, Germany

${ }^{3}$ School of Geography, Earth and Atmospheric Sciences, University of Melbourne, Australia

\section{CONTACT}

Kira Rehfeld: kira.rehfeld@uni-tuebingen.de Josephine Brown: josephine.brown@unimelb.edu.au

\section{REFERENCES}

An S-I, Choi J (2014) Monthly Weather Review 43: $957-970$ Braconnot P et al. (2012) Nat Clim Chang 2: 417-424

Brown JR et al. (2020) Clim Past 16: 1777-1805

Emile-Geay J et al. (2016) Nat Geosci 9: 168-173

Jungclaus JH et al. (2017) Geosci Model Dev 10: 4005-4033

Kageyama M et al. (2018) Geosci Model Dev 11: 1033-1057

Laepple T, Huybers P (2014) Proc Natl Acad Sci USA 111: 16682-16687

Masson-Delmotte V et al. (2013) In: Stocker TF et al. (Eds) Climate Change 2013: The Physical Science Basis. Cambridge University Press, 383-464

PAGES 2k Consortium (2019) Nat Geosci 12: 643-649

Parsons LA et al. (2020) Geophys Res Lett 47: e2019GL086588

Rehfeld K et al. (2018) Nature 554: 356-359

Rehfeld K et al. (2020) Earth Syst Dyn 11: 447-468

Saint-Lu M et al. (2015) Earth Planet Sci Lett 412: 18-24

Taylor KE et al. (2012) Bull Am Meteorol Soc 93: 485-498 Zheng W et al. (2008) Clim Dyn 30: 745-762 